\title{
Eigenvalues of a Class of Eigenparameter Dependent Third-Order Differential Operators
}

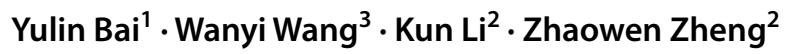

Received: 25 October 2021 / Accepted: 20 January 2022 / Published online: 21 February 2022

(C) The Author(s) 2022

\begin{abstract}
The present paper deals with a class of third-order differential operators with eigenparameter dependent boundary conditions. The continuity of eigenvalues concerning a given parameter is proved. Moreover, the derivative formulas of eigenvalues concerning the parameters, in particular, the eigenparameter dependent boundary condition matrix, are given.
\end{abstract}

Keywords Third-order differential operators · Eigenparameter dependent boundary conditions $\cdot$ Continuity of eigenvalues $\cdot$ Differentiability of eigenvalues

\section{Introduction}

Boundary value problem (BVP) with an eigenparameter contained in equations and boundary conditions is a significant part of differential operator theory for its wide applications in various fields. And it has attracted more and more attentions from mathematicians and physicists in recent decades. Various applications in physics and other fields such as the vibration of loaded strings, diffusion processes in probability theory and so on yield such problems [1]. A large number of literature have devoted to the study of such problems for Sturm-Liouville (S-L) problems and fourth-order beam equations, and numerous significance results are obtained (see, for example [2-12]).

The dependence of eigenvalues of classical regular S-L problems has been well investigated by Kong, $\mathrm{Wu}$ and Zettl. They showed that each eigenvalue of regular $\mathrm{S}-\mathrm{L}$ problems can be inserted into a continuous eigenvalue branch, moreover, it is

Kun Li

qslikun@163.com

1 College of Mathematics Science, Inner Mongolia Normal University, Hohhot 010022, China

2 School of Mathematical Sciences, Qufu Normal University, Qufu 273165, China

3 School of Mathematical Sciences, Inner Mongolia Agricultural University, Hohhot 010018, China 
shown that each eigenvalue is differentiable with regards to a given parameter [13, 14] (the coefficients of differential expression, the endpoints and the boundary conditions). Such dependence has been generalized by Shi, Zettl etc. into singular S-L problems, especially in the case of limit point $S-L$ problems $[15,16]$. The dependence of eigenvalues of eigenparameter dependent S-L problems was investigated by Zhang etc. [17]. And in [18-20] fourth-order and higher-order differential operators were studied. In [21-24], the dependence of eigenvalues of discontinuous differential operators were investigated. These results play a significant role in the eigenvalue theory of differential operators and it is fundamental from the numerical computation of spectrum, for example, the codes SLEIGN2 [25] and SLEUTH [26].

Boundary condition plays a crucial role in the differential operators theory since it carries the information of the spectrum. As is well-known, the self-adjoint boundary conditions of differential operators can be classified into three cases: mixed, separated and coupled, which was shown by Wang, Sun and Zettl [27]. For the second-order S-L operators, there only exist two cases: separated and coupled. However, with regard to third-order differential operators, there only coupled and mixed self-adjoint boundary conditions exist [28] and a complete characterization of self-adjoint domain of odd order differential operators was given in [29]. Also, third-order differential equations have numerous physical backgrounds, for example, three-layer beam, the deflection of a curved beam varying cross-section, and so on $[30,31]$. Based on these facts, Uğurlu investigated the continuity and differentiability of eigenvalues concerning some data for third-order self-adjoint differential operators without eigenparameter dependent boundary conditions, moreover, the differential expressions of eigenvalues concerning some parameters are given [32]. Therefore, a natural question is rising: does a small change of third-order differential operators with eigenparameter dependent boundary conditions results in small change of each eigenvalue and eigenfunction of the problem? In the present paper, we will solve this problem.

In this paper, we will consider a class of eigenparameter dependent third-order differential operators with mixed boundary conditions. We characterize the continuity of eigenvalues with respect to given data by using the continuity theorem for analytic functions with isolated zeros, moreover, the differential expressions of eigenvalues are also presented. The paper is organized as follows. In Sect. 2, we investigate some notations and preliminaries associated with the problem. The continuity and differentiability of eigenvalues are presented in Sects. 3 and 4, respectively.

\section{Preliminaries}

We study third-order symmetric differential equation

$$
\ell f:=\frac{1}{w}\left\{-i\left[q_{0}\left(q_{0} f^{\prime}\right)^{\prime}\right]^{\prime}-\left(p_{0} f^{\prime}\right)^{\prime}+i\left[q_{1} f^{\prime}+\left(q_{1} f\right)^{\prime}\right]+p_{1} f\right\}=\lambda f, \quad \text { on }[a, b],
$$

with boundary conditions: 


$$
\begin{gathered}
L_{1} f:=\left(\alpha_{1} \lambda+\widetilde{\alpha}_{1}\right) f(a)-\left(\alpha_{2} \lambda+\widetilde{\alpha}_{2}\right) f^{[2]}(a)=0, \\
L_{2} f:=\left(\gamma_{1} \lambda+\widetilde{\gamma}_{1}\right) f(b)+\left(\gamma_{2} \lambda+\widetilde{\gamma}_{2}\right) f^{[2]}(b)=0, \\
L_{3} f:=(\sin \theta+i) f^{[1]}(a)+(i \sin \theta+1) f^{[1]}(b)=0,
\end{gathered}
$$

where $\lambda$ is the spectral parameter, $\theta \in(0, \pi], q_{0}, q_{1}, p_{0}, p_{1}, w$ satisfy the conditions

$$
q_{0}^{-1}, q_{0}^{-2}, p_{0}, q_{1}, p_{1}, w \in L^{1}([a, b], \mathbb{R}), \quad q_{0}>0, \quad w>0 .
$$

$\alpha_{k}, \tilde{\alpha}_{k}, \gamma_{k}, \tilde{\gamma}_{k}(k=1,2)$ are arbitrary real numbers, and satisfy

$$
\rho_{1}=\tilde{\alpha}_{1} \alpha_{2}-\alpha_{1} \tilde{\alpha}_{2}>0, \quad \rho_{2}=\tilde{\gamma}_{1} \gamma_{2}-\gamma_{1} \tilde{\gamma}_{2}>0 .
$$

Third-order BVP with an eigenparameter contained in the boundary conditions consisting of (1)-(4) is considered here. Firstly, we investigate some basic preliminaries.

The quasi-derivatives of $f$ are defined as [33]

$$
f^{[0]}=f, f^{[1]}=-\frac{1+i}{\sqrt{2}} q_{0} f^{\prime}, \quad f^{[2]}=i q_{0}\left(q_{0} f^{\prime}\right)^{\prime}+p_{0} f^{\prime}-i q_{1} f .
$$

and $H_{w}=L^{2}[a, b]$ is a weighted Hilbert space equipped with the inner product $\langle f, g\rangle_{w}=\int_{a}^{b^{w}} f \bar{g} w d x$ consisting of functions $f$ which satisfy $\int_{a}^{b}|f|^{2} w d x<\infty$.

Maximal operator $L_{\max }$ is defined as

$$
L_{\max } f=\ell f, \quad f \in H_{w}
$$

with the domain

$$
D_{\max }=\left\{f \in L_{w}^{2}[a, b] \mid f, f^{[1]}, f^{[2]} \in A C[a, b], \ell f \in L_{w}^{2}[a, b]\right\} .
$$

Then for arbitrary $f, g \in D_{\max }$, integration by parts yields Lagrange identity

$$
\left\langle L_{\max } f, g\right\rangle_{w}-\left\langle f, L_{\max } g\right\rangle_{w}=[f, \bar{g}]_{a}^{b},
$$

where

$$
\begin{aligned}
& {[f, \bar{g}]_{a}^{b}=[f, \bar{g}](b)-[f, \bar{g}](a),} \\
& {[f, \bar{g}](x)=f(x) \overline{g^{[2]}(x)}-f^{[2]}(x) \overline{g(x)}+i f^{[1]}(x) \overline{g^{[1]}(x)} .}
\end{aligned}
$$

Let $\mathcal{H}=L_{w}^{2}[a, b] \oplus \mathbb{C}^{2}$ equipped with inner product

$$
\langle F, G\rangle=\int_{a}^{b} f \bar{g} w d x+\frac{1}{\rho_{1}} f_{1} \overline{g_{1}}+\frac{1}{\rho_{2}} f_{2} \overline{g_{2}},
$$

where $F=\left(\begin{array}{c}f(x) \\ f_{1} \\ f_{2}\end{array}\right), G=\left(\begin{array}{c}g(x) \\ g_{1} \\ g_{2}\end{array}\right) \in \mathcal{H}$. It is easy verified that $\mathcal{H}$ is a Hilbert space. 
Define the operator $\mathrm{T}$ as follows

$$
\begin{aligned}
D(\mathrm{~T}) & =\left\{F=\left(f(x), f_{1}, f_{2}\right)^{T} \in \mathcal{H} \mid L_{3} f=0,\right. \\
f_{1} & \left.=\alpha_{1} f(a)-\alpha_{2} f^{[2]}(a), f_{2}=\gamma_{1} y(b)+\gamma_{2} f^{[2]}(b), f \in D_{\max }\right\},
\end{aligned}
$$

and

$$
F(x)=\left(\begin{array}{c}
f(x) \\
\alpha_{1} f(a)-\alpha_{2} f^{[2]}(a) \\
\gamma_{1} f(b)+\gamma_{2} f^{[2]}(b)
\end{array}\right) \in D(\mathrm{~T}), \quad \mathrm{T} F=\left(\begin{array}{c}
\ell f \\
\widetilde{\alpha}_{2} f^{[2]}(a)-\widetilde{\alpha}_{1} f(a) \\
-\left[\widetilde{\gamma}_{1} f(b)+\widetilde{\gamma}_{2} f^{[2]}(b)\right]
\end{array}\right) .
$$

By the definition of the operator T, the eigenvalue problem of BVP (1)-(4) is transferred to the spectra problem of the operator $\mathrm{T}$.

Considering the operator $\mathrm{T}$, the following properties hold.

Lemma 2.1 $\mathrm{T}$ is self-adjoint in $\mathcal{H}$, its eigenvalues are discrete, real-valued and have no finite point of accumulation. Moreover, the multiplicity of each eigenvalue at most 3 .

Proof For detail proof, we refer to [34].

Let

$$
\begin{aligned}
A_{\lambda} & =\left(\begin{array}{ccc}
\alpha_{1} \lambda+\widetilde{\alpha}_{1} & 0 & -\left(\alpha_{2} \lambda+\widetilde{\alpha}_{2}\right) \\
0 & 0 & 0 \\
0 & \sin \theta+i & 0
\end{array}\right), \\
B_{\lambda} & =\left(\begin{array}{ccc}
0 & 0 & 0 \\
\gamma_{1} \lambda+\tilde{\gamma}_{1} & 0 & \gamma_{2} \lambda+\tilde{\gamma}_{2} \\
0 & i \sin \theta+1 & 0
\end{array}\right) .
\end{aligned}
$$

Let $\vartheta_{1}(x, \lambda), \vartheta_{2}(x, \lambda), \vartheta_{3}(x, \lambda)$ be the system of linearly independent fundamental solutions of Eq. (1) satisfy initial condition

$$
\Theta(x, \lambda)=\left(\begin{array}{lll}
\vartheta_{1}^{[0]}(x, \lambda) & \vartheta_{2}^{[0]}(x, \lambda) & \vartheta_{3}^{[0]}(x, \lambda) \\
\vartheta_{1}^{[1]}(x, \lambda) & \vartheta_{2}^{[1]}(x, \lambda) & \vartheta_{3}^{[1]}(x, \lambda) \\
\vartheta_{1}^{[2]}(x, \lambda) & \vartheta_{2}^{[2]}(x, \lambda) & \vartheta_{3}^{[2]}(x, \lambda)
\end{array}\right)=\left(\begin{array}{lll}
1 & 0 & 0 \\
0 & 1 & 0 \\
0 & 0 & 1
\end{array}\right) .
$$

Lemma $2.2 \lambda \in \mathbb{C}$ is an eigenvalue of BVP (1)-(4) (also of the operator $\mathrm{T}$ ) if and only if

$$
\Delta(\lambda)=\operatorname{det}\left[A_{\lambda}+B_{\lambda} \Theta(b, \lambda)\right]=0 .
$$

Proof The proof is routine by substituting the general solution consisting of the independent solutions $\vartheta_{1}(x, \lambda), \vartheta_{2}(x, \lambda), \vartheta_{3}(x, \lambda)$ into boundary conditions (2)-(4), 
then we can get a system of homogeneous linear equations, using ordinary differential equation theory, we can get the conclusion.

\section{The Banach Space}

For purpose of investigating the continuity of eigenvalues, we introduce the following Banach space.

Consider the Banach space

$$
\mathfrak{W}=L^{1}[a, b] \oplus L^{1}[a, b] \oplus L^{1}[a, b] \oplus \mathbb{R}^{9}
$$

with the norm

$$
\|\omega\|=\int_{a}^{b}\left(\left|p_{0}\right|+\left|p_{1}\right|+|w|\right) d x+\left|\Delta_{1}\right|+\left|\Delta_{2}\right|+|\theta|
$$

for any $W=\left(p_{0}, p_{1}, w, \Delta_{1}, \Delta_{2}, \theta\right) \in \mathfrak{B}$, where

$$
\left|\Delta_{1}\right|=\left|\alpha_{1}\right|+\left|\alpha_{2}\right|+\left|\widetilde{\alpha}_{1}\right|+\left|\widetilde{\alpha}_{2}\right|, \quad\left|\Delta_{2}\right|=\left|\gamma_{1}\right|+\left|\gamma_{2}\right|+\left|\widetilde{\gamma}_{1}\right|+\left|\tilde{\gamma}_{2}\right| .
$$

Let

$$
\Omega=\{\omega \in \mathfrak{W}:(2.5),(2.6) \text { hold, and } \theta \in(0, \pi]\} .
$$

Lemma 3.1 The solution $f=f\left(x, c_{0}, d_{1}, d_{2}, d_{3}, p_{0}, p_{1}, w\right)$ of Eq. (1) satisfying the conditions

$$
f\left(c_{0}, \lambda\right)=d_{1}, f^{[1]}\left(c_{0}, \lambda\right)=d_{2}, f^{[2]}\left(c_{0}, \lambda\right)=d_{3}, c_{0} \in[a, b]
$$

is continuous with respect to all its variables.

Proof By transferring the Eq. (1) to a first-order system, and based on the existence and uniqueness of solutions and Theorem 2.7 in [35], the conclusion holds.

Theorem 3.2 Let $\omega^{*}=\left(p_{0}^{*}, p_{1}^{*}, w^{*}, \Delta_{1}^{*}, \Delta_{2}^{*}, \theta^{*}\right) \in \Omega$, and $\lambda=\lambda(\omega)$ be an eigenvalue of BVP (1)-(4). Then for any $\varepsilon>0$, there exists a $\delta>0$ so that if $\omega \in \Omega$ satisfies

$$
\begin{aligned}
\left\|\omega-\omega^{*}\right\|= & \int_{a}^{b}\left(\left|p_{0}-p_{0}^{*}\right|+\left|p_{1}-p_{1}^{*}\right|+\left|w-w^{*}\right|\right) d x \\
& +\left|\alpha_{1}-\alpha_{1}^{*}\right|+\left|\alpha_{2}-\alpha_{2}^{*}\right|+\left|\widetilde{\alpha}_{1}-\widetilde{\alpha}_{1}^{*}\right|+\left|\widetilde{\alpha}_{2}-\widetilde{\alpha}_{2}^{*}\right| \\
& +\left|\gamma_{1}-\gamma_{1}^{*}\right|+\left|\gamma_{2}-\gamma_{2}^{*}\right|+\left|\widetilde{\gamma}_{1}-\widetilde{\gamma}_{1}^{*}\right|+\left|\widetilde{\gamma}_{2}-\widetilde{\gamma}_{2}^{*}\right|+\left|\theta-\theta^{*}\right| \\
& <\delta,
\end{aligned}
$$

then

$$
\left|\lambda(\omega)-\lambda\left(\omega^{*}\right)\right|<\varepsilon
$$


Proof By Lemma 2.2, one can see that $\lambda$ is an eigenvalue of BVP (1)-(4) if and only if $\Delta(\lambda)=0$. By the continuous dependence of solutions on the problem ([36], Theorem 2.4.1), $\Delta(\lambda)$ is an entire function with respect to $\lambda$ and is continuous at $\omega \in \mathfrak{W}$. We can also verified that $\Delta(\lambda)$ is not a constant. According to the continuity theorem for analytic functions with isolated zeros, the conclusion holds.

Definition 1 We call $F=\left(f(x), f_{1}, f_{2}\right)^{T} \in H$ is an normalized eigenvector of the operator T, where $f_{1}=\alpha_{1} f(a)-\alpha_{2} f^{[2]}(a), f_{2}=\gamma_{1} f(b)+\gamma_{2} f^{[2]}(b)$, if the eigenvector $F=\left(f(x), f_{1}, f_{2}\right)^{T}$ of T satisfies

$$
\begin{aligned}
\|F\|^{2} & =\left\langle\left(f(x), f_{1}, f_{2}\right)^{T},\left(f(x), f_{1}, f_{2}\right)^{T}\right\rangle \\
& =\int_{a}^{b}|f(x)|^{2} w d x+\frac{1}{\rho_{1}}\left|f_{1}\right|^{2}+\frac{1}{\rho_{2}}\left|f_{2}\right|^{2} \\
& =1 .
\end{aligned}
$$

The continuity of eigenvectors of the operator $\mathrm{T}$ is stated as follows.

Theorem 3.3 Let $\lambda(\omega)(\omega \in \Omega)$ be an eigenvalue with multiplicity $n(n=1,2,3)$ for all $\omega$ in some neighborhoods of $\omega^{*}$ in $\Omega$. Let $F_{k}\left(x, \omega^{*}\right)=\left(f_{k}\left(x, \omega^{*}\right), f_{k 1}\left(\omega^{*}\right), f_{k 2}\left(\omega^{*}\right)\right)^{T}$ be the normalized eigenvectors of $\lambda\left(\omega^{*}\right)$. Then, there exist $n$ linearly independent normalized eigenvectors $F_{k}(x, \omega)=\left(f_{k}(x, \omega), f_{k 1}(\omega), f_{k 2}(\omega)\right)^{T}$ of $\lambda(\omega)$ so that

$$
\begin{aligned}
& f_{k}(x, \omega) \rightarrow f_{k}\left(x, \omega^{*}\right), f_{k}^{[1]}(x, \omega) \rightarrow f_{k}^{[1]}\left(x, \omega^{*}\right), f_{k}^{[2]}(x, \omega) \rightarrow f_{k}^{[2]}\left(x, \omega^{*}\right) \\
& f_{k 1}(\omega) \rightarrow f_{k 1}\left(\omega^{*}\right), f_{k 2}(\omega) \rightarrow f_{k 2}\left(\omega^{*}\right), \quad k=1, \ldots, n, \text { as } \omega \rightarrow \omega^{*} \quad \text { in } \Omega
\end{aligned}
$$

both uniformly on $[a, b]$.

Proof It can be proved by using similar methods in [17], with the aid of Lemma 3.1, Theorem 3.2 as well as Theorem 3.2 in [13].

\section{Derivative Formulas of Eigenvalues}

We give the differentiability of eigenvalues concerning some data, especially the boundary condition parameter matrix in this section.

Definition $2{ }^{[12]}$ Let $\mathbf{X}, \mathbf{Y}$ be Banach spaces. We call the map $\Gamma: \mathbf{X} \rightarrow \mathbf{Y}$ is Fréchet differentiable at $x \in \mathbf{X}$ provided that a bounded linear operator $\mathrm{d} \Gamma_{x}: \mathbf{X} \rightarrow \mathbf{Y}$ exists, such that for $\tau \in \mathbf{X}$,

$$
\left|\Gamma(x+\tau)-\Gamma(x)-\mathrm{d} \Gamma_{x}(\tau)\right|=o(\tau), \quad \tau \rightarrow 0 .
$$

Theorem 4.1 Let $\omega=\left(p_{0}, p_{1}, w, \Delta_{1}, \Delta_{2}, \theta\right) \in \Omega, \lambda=\lambda(\omega)$ be an eigenvalue of $B V P$ (1)-(4), $F(\omega)=\left(f(x, \omega), f_{1}(\omega), f_{2}(\omega)\right)^{T^{2}} \in \mathcal{H}$ be the corresponding eigenvector. $E$ is the identity matrix, $R$ is $2 \times 2$ real-valued matrix. Assume that for all fixed elements 
of $\omega$ except one, the geometric multiplicity of $\lambda(\omega)$ is invariant in some neighborhoods $\mathcal{M} \subset \Omega$. Then the following differential expressions hold.

(1) Let all the elements of $\omega$ be fixed except $\theta$ and $\lambda=\lambda(\theta)$. Then $\lambda$ is differentiable and satisfies

$$
\lambda^{\prime}(\theta)=\frac{2 \cos \theta}{1+\sin ^{2} \theta}\left|f^{[1]}(a)\right|^{2}=\frac{2 \cos \theta}{1+\sin ^{2} \theta}\left|f^{[1]}(b)\right|^{2} .
$$

(2) Let all the elements of $\omega$ be fixed except $\alpha_{1}$ and $\lambda=\lambda\left(\alpha_{1}\right)$. Then $\lambda$ is differentiable and satisfies

$$
\lambda^{\prime}\left(\alpha_{1}\right)=\frac{\lambda}{\alpha_{2} \lambda+\widetilde{\alpha}_{2}}|f(a)|^{2},
$$

where $\alpha_{2} \lambda+\widetilde{\alpha}_{2} \neq 0$.

(3) Let all the elements of $\omega$ be fixed except $\widetilde{\alpha}_{1}$ and $\lambda=\lambda\left(\widetilde{\alpha}_{1}\right)$. Then $\lambda$ is differentiable and satisfies

$$
\lambda^{\prime}\left(\widetilde{\alpha}_{1}\right)=\frac{1}{\alpha_{2} \lambda+\widetilde{\alpha}_{2}}|f(a)|^{2},
$$

where $\alpha_{2} \lambda+\widetilde{\alpha}_{2} \neq 0$.

(4) Let all the elements of $\omega$ be fixed except $\alpha_{2}$ and $\lambda=\lambda\left(\alpha_{2}\right)$. Then $\lambda$ is differentiable and satisfies

$$
\lambda^{\prime}\left(\alpha_{2}\right)=-\frac{\lambda}{\alpha_{1} \lambda+\widetilde{\alpha}_{1}}\left|f^{[2]}(a)\right|^{2},
$$

where $\alpha_{1} \lambda+\widetilde{\alpha}_{1} \neq 0$.

(5) Let all the elements of $\omega$ be fixed except $\widetilde{\alpha}_{2}$ and $\lambda=\lambda\left(\widetilde{\alpha}_{2}\right)$. Then $\lambda$ is differentiable and satisfies

$$
\lambda^{\prime}\left(\widetilde{\alpha}_{2}\right)=-\frac{1}{\alpha_{1} \lambda+\widetilde{\alpha}_{1}}\left|f^{[2]}(a)\right|^{2},
$$

where $\alpha_{1} \lambda+\widetilde{\alpha}_{1} \neq 0$.

(6) Let all the elements of $\omega$ be fixed except $\gamma_{1}$ and $\lambda=\lambda\left(\gamma_{1}\right)$. Then $\lambda$ is differentiable and satisfies

$$
\lambda^{\prime}\left(\gamma_{1}\right)=\frac{\lambda}{\gamma_{2} \lambda+\widetilde{\gamma}_{2}}|f(b)|^{2},
$$

where $\gamma_{2} \lambda+\tilde{\gamma}_{2} \neq 0$.

(7) Let all the elements of $\omega$ be fixed except $\tilde{\gamma}_{1}$ and $\lambda=\lambda\left(\widetilde{\gamma}_{1}\right)$. Then $\lambda$ is differentiable and satisfies 


$$
\lambda^{\prime}\left(\widetilde{\gamma}_{1}\right)=\frac{1}{\gamma_{2} \lambda+\widetilde{\gamma}_{2}}|f(b)|^{2}
$$

where $\gamma_{2} \lambda+\widetilde{\gamma}_{2} \neq 0$.

(8) Let all the elements of $\omega$ be fixed except $\gamma_{2}$ and $\lambda=\lambda\left(\gamma_{2}\right)$. Then $\lambda$ is differentiable and satisfies

$$
\lambda^{\prime}\left(\gamma_{2}\right)=-\frac{\lambda}{\gamma_{1} \lambda+\widetilde{\gamma}_{1}}\left|f^{[2]}(b)\right|^{2},
$$

where $\gamma_{1} \lambda+\widetilde{\gamma}_{1} \neq 0$.

(9) Let all the elements of $\omega$ be fixed except $\tilde{\gamma}_{2}$ and $\lambda=\lambda\left(\widetilde{\gamma}_{2}\right)$. Then $\lambda$ is differentiable and satisfies

$$
\lambda^{\prime}\left(\widetilde{\gamma}_{2}\right)=-\frac{1}{\gamma_{1} \lambda+\widetilde{\gamma}_{1}}\left|f^{[2]}(b)\right|^{2},
$$

where $\gamma_{1} \lambda+\tilde{\gamma}_{1} \neq 0$.

(10) Let all the elements of $\omega$ be fixed except the boundary condition parameter matrix $K=\left(\begin{array}{cc}\alpha_{1} & \widetilde{\alpha}_{1} \\ \alpha_{2} & \widetilde{\alpha}_{2}\end{array}\right)$ and $\lambda=\lambda(K)$. Then for all $R$ satisfying $\operatorname{det}[K+R]=-\rho_{1}$, the Fréchet derivative of $\lambda$ with respect to $K$ is formulated as

$d \lambda_{K}(R)=\left(-f(a), f^{[2]}(a)\right)\left[E-(K+R) K^{-1}\right]\left(\overline{f^{[2]}(a)}, \overline{f(a)}\right)^{T}$.

(11) Let all the elements of $\omega$ be fixed except the boundary condition parameter matrix $\widetilde{K}=\left(\begin{array}{ll}\gamma_{1} & \widetilde{\gamma}_{1} \\ \gamma_{2} & \widetilde{\gamma}_{2}\end{array}\right)$ and $\lambda=\lambda(\widetilde{K})$. Then for all $R$ satisfying $\operatorname{det}[\widetilde{K}+R]=-\rho_{2}$, the Fréchet derivative of $\lambda$ with respect to $\widetilde{K}$ is formulated as

$d \lambda_{\widetilde{K}}(R)=\left(f(b), f^{[2]}(b)\right)\left[E-(\widetilde{K}+R) \widetilde{K}^{-1}\right]\left(\overline{f^{[2]}(b)},-\overline{f(b)}\right)^{T}$.

(12) Let all the elements of $\omega$ be fixed except $p_{0}$ and $\lambda=\lambda\left(p_{0}\right)$. Then the Fréchet derivative of $\lambda$ with respect to $p_{0}$ is formulated as

$$
d \lambda_{p_{0}}(\tau)=\int_{a}^{b} \tau\left|f^{[1]}\right|^{2} d x, \quad \tau \in L^{1}[a, b] .
$$

(13) Let all the elements of $\omega$ be fixed except $p_{1}$ and $\lambda=\lambda\left(p_{1}\right)$. Then the Fréchet derivative of $\lambda$ with respect to $p_{1}$ is formulated as

$$
d \lambda_{p_{1}}(\tau)=\int_{a}^{b} \tau|f|^{2} d x, \quad \tau \in L^{1}[a, b] .
$$


(14) Let all the elements of $\omega$ be fixed except $w$ and $\lambda=\lambda(w)$. Then the Fréchet derivative of $\lambda$ with respect to $w$ is formulated as

$$
d \lambda_{w}(\tau)=\lambda_{w} \int_{a}^{b} \tau|f|^{2} d x, \quad \tau \in L^{1}[a, b] .
$$

Proof Let all the elements of $\omega \in \Omega$ be fixed except one. For sufficiently small $\varepsilon>0$, when $\left\|\omega-\omega^{*}\right\|<\varepsilon$, let $\lambda(\omega)$ be an eigenvalue of BVP (1)-(4) satisfying Theorem 3.2. For all the above six cases, the $\lambda(\omega)$ is replaced by $\lambda(\theta+\Delta \theta), \lambda\left(\alpha_{1}+\Delta \alpha_{1}\right)$, $\lambda\left(\widetilde{\alpha}_{1}+\Delta \widetilde{\alpha}_{1}\right), \quad \lambda\left(\alpha_{2}+\Delta \alpha_{2}\right), \quad \lambda\left(\widetilde{\alpha}_{2}+\Delta \widetilde{\alpha}_{2}\right), \quad \lambda\left(\gamma_{1}+\Delta \gamma_{1}\right), \quad \lambda\left(\widetilde{\gamma}_{1}+\Delta \tilde{\gamma}_{1}\right), \quad \lambda\left(\gamma_{2}+\Delta \gamma_{2}\right)$, $\lambda\left(\widetilde{\gamma}_{2}+\Delta \tilde{\gamma}_{2}\right), \lambda(K+R), \lambda(\widetilde{K}+R), \lambda\left(p_{0}+\eta\right), \lambda\left(p_{1}+\eta\right), \lambda(w+\eta)$, respectively.

(1) Let all the elements of $\omega$ be fixed except $\theta$. We adopt the following notations:

$$
\begin{array}{ll}
\mathcal{B}_{1}(f)=\alpha_{1} f(a)-\alpha_{2} f^{[2]}(a), & \mathcal{B}_{2}(f)=\gamma_{1} f(b)+\gamma_{2} f^{[2]}(b), \\
\mathcal{D}_{1}(f)=\widetilde{\alpha}_{2} f^{[2]}(a)-\widetilde{\alpha}_{1} f(a), & \mathcal{D}_{2}(f)=-\left[\widetilde{\gamma}_{1} f(b)+\widetilde{\gamma}_{2} f^{[2]}(b)\right] .
\end{array}
$$

Let

$$
\begin{aligned}
& F(x, \theta)=\left(f(x, \theta), f_{1}(\theta), f_{2}(\theta)\right)^{T} \\
& G(x, \theta)=\left(g(x, \theta), g_{1}(\theta), g_{2}(\theta)\right)^{T}
\end{aligned}
$$

be the normalized eigenvectors corresponding to $\lambda(\theta)$ and $\lambda(\theta+\Delta \theta)$ respectively. By the self-adjointness of the operator T and the boundary condition (4), we have

$$
\begin{aligned}
& {[\lambda(\theta+\Delta \theta)-\lambda(\theta)]\langle G, F\rangle} \\
& \quad=\langle\lambda(\theta+\Delta \theta) G, F\rangle-\langle G, \lambda(\theta) F\rangle \\
& \quad=[g, \bar{f}]_{a}^{b}+\frac{1}{\rho_{1}}\left[\mathcal{D}_{1}(g) \overline{\mathcal{B}_{1}(f)}-\mathcal{B}_{1}(g) \overline{\mathcal{D}_{1}(f)}\right]+\frac{1}{\rho_{2}}\left[\mathcal{D}_{2}(g) \overline{\mathcal{B}_{2}(f)}-\mathcal{B}_{2}(g) \overline{\mathcal{D}_{2}(f)}\right] \\
& \quad=i g^{[1]}(b) \overline{f^{[1]}(b)}-i g^{[1]}(a) \overline{f^{[1]}(a)} \\
& \quad=i\left[\frac{i+\sin (\theta+\Delta \theta)}{1+i \sin (\theta+\Delta \theta)} \frac{\sin \theta-i}{1-i \sin \theta}-1\right] g^{[1]}(a) \overline{f^{[1]}(a)}
\end{aligned}
$$

Dividing both sides of $\Delta \theta$, and taking the limit as $\Delta \theta \rightarrow 0$, we have

$$
\lambda^{\prime}(\theta)=\frac{2 \cos \theta}{1+\sin ^{2} \theta}\left|f^{[1]}(a)\right|^{2}
$$

by Theorem 3.3. Using the boundary condition (4), we have $\left|f^{[1]}(a)\right|^{2}=\left|f^{[1]}(b)\right|^{2}$. Hence, (1) holds.

(2) Let all the elements of $\omega$ be fixed except $\alpha_{1}$, and 


$$
\begin{aligned}
& F\left(x, \alpha_{1}\right)=\left(f\left(x, \alpha_{1}\right), f_{1}\left(\alpha_{1}\right), f_{2}\left(\alpha_{1}\right)\right)^{T}, \\
& G\left(x, \alpha_{1}\right)=\left(g\left(x, \alpha_{1}\right), g_{1}\left(\alpha_{1}\right), g_{2}\left(\alpha_{1}\right)\right)^{T}
\end{aligned}
$$

be the normalized eigenvectors corresponding to $\lambda\left(\alpha_{1}\right)$ and $\lambda\left(\alpha_{1}+\Delta \alpha_{1}\right)$, respectively. By the self-adjointness of the operator T and the boundary condition (2), (4), we have

$$
\begin{aligned}
{[\lambda(} & \left.\left.\alpha_{1}+\Delta \alpha_{1}\right)-\lambda\left(\alpha_{1}\right)\right]\langle G, F\rangle \\
= & \left\langle\lambda\left(\alpha_{1}+\Delta \alpha_{1}\right) G, F\right\rangle-\left\langle G, \lambda\left(\alpha_{1}\right) F\right\rangle \\
= & {[g, \bar{f}]_{a}^{b}+\frac{1}{\rho_{1}}\left[\mathcal{D}_{1}(g) \overline{\mathcal{B}_{1}(f)}-\mathcal{B}_{1}(g) \overline{\mathcal{D}_{1}(f)}\right]+\frac{1}{\rho_{2}}\left[\mathcal{D}_{2}(g) \overline{\mathcal{B}_{2}(f)}-\mathcal{B}_{2}(g) \overline{\mathcal{D}_{2}(f)}\right] } \\
= & g^{[2]}(a) \overline{f(a)}-g(a) \overline{f^{[2]}(a)}+\frac{1}{\rho_{1}}\left[\widetilde{\alpha}_{2} g^{[2]}(a)-\widetilde{\alpha}_{1} g(a)\right]\left[\alpha_{1} \overline{f(a)}-\alpha_{2} \overline{f^{[2]}(a)}\right] \\
& -\frac{1}{\rho_{1}}\left[\left(\alpha_{1}+\Delta \alpha_{1}\right) g(a)-\alpha_{2} g^{[2]}(a)\right]\left[\widetilde{\alpha}_{2} \overline{f^{[2]}(a)}-\widetilde{\alpha}_{1} \overline{f(a)}\right] \\
= & \frac{\Delta \alpha_{1}}{\rho_{1}}\left(\widetilde{\alpha}_{1} g(a) \overline{f(a)}-\widetilde{\alpha}_{2} g(a) \bar{f} \overline{f^{[2]}(a)}\right) \\
= & \frac{\Delta \alpha_{1}}{\rho_{1}}\left(\widetilde{\alpha}_{1}-\widetilde{\alpha}_{2} \frac{\alpha_{1} \lambda+\widetilde{\alpha}_{1}}{\alpha_{2} \lambda+\widetilde{\alpha}_{2}}\right) g(a) \overline{f(a)} \\
= & \frac{\lambda \Delta \alpha_{1}}{\alpha_{2} \lambda+\widetilde{\alpha}_{2}} g(a) \overline{f(a)}
\end{aligned}
$$

Dividing both sides of $\Delta \alpha_{1}$, and taking the limit as $\Delta \alpha_{1} \rightarrow 0$, we have

$$
\lambda^{\prime}\left(\alpha_{1}\right)=\frac{\lambda}{\alpha_{2} \lambda+\widetilde{\alpha}_{2}}|f(a)|^{2}
$$

by Theorem 3.3. Using the same methods of (2), one can prove that (3), (4) and (5) are also true.

(6) Let all the elements of $\omega$ be fixed except $\gamma_{1}$, and

$$
\begin{aligned}
& F\left(x, \gamma_{1}\right)=\left(f\left(x, \gamma_{1}\right), f_{1}\left(\gamma_{1}\right), f_{2}\left(\gamma_{1}\right)\right)^{T}, \\
& G\left(x, \gamma_{1}\right)=\left(g\left(x, \gamma_{1}\right), g_{1}\left(\gamma_{1}\right), g_{2}\left(\gamma_{1}\right)\right)^{T}
\end{aligned}
$$

be the normalized eigenvectors corresponding to $\lambda\left(\gamma_{1}\right)$ and $\lambda\left(\gamma_{1}+\Delta \gamma_{1}\right)$, respectively. Then By the self-adjointness of the operator $\mathrm{T}$ and the boundary condition (3), (4), we have 


$$
\begin{aligned}
{\left[\lambda \left(\gamma_{1}\right.\right.} & \left.\left.+\Delta \gamma_{1}\right)-\lambda\left(\gamma_{1}\right)\right]\langle G, F\rangle \\
= & \left\langle\lambda\left(\gamma_{1}+\Delta \gamma_{1}\right) G, F\right\rangle-\left\langle G, \lambda\left(\gamma_{1}\right) F\right\rangle \\
= & {[g, \bar{f}]_{a}^{b}+\frac{1}{\rho_{1}}\left[\mathcal{D}_{1}(g) \overline{\mathcal{B}_{1}(f)}-\mathcal{B}_{1}(g) \overline{\mathcal{D}_{1}(f)}\right]+\frac{1}{\rho_{2}}\left[\mathcal{D}_{2}(g) \overline{\mathcal{B}_{2}(f)}-\mathcal{B}_{2}(g) \overline{\mathcal{D}_{2}(f)}\right] } \\
= & g(b) \overline{f^{[2]}(b)}-g^{[2]}(b) \overline{f(b)}-\frac{1}{\rho_{2}}\left[\widetilde{\gamma}_{1} g(b)+\widetilde{\gamma}_{2} g^{[2]}(b)\right]\left[\gamma_{1} \overline{f(b)}+\gamma_{2} \overline{f^{[2]}(b)}\right] \\
& +\frac{1}{\rho_{2}}\left[\left(\gamma_{1}+\Delta \gamma_{1}\right) g(b)+\gamma_{2} g^{[2]}(b)\right]\left[\widetilde{\gamma}_{1} \overline{f(b)}+\widetilde{\gamma}_{2} \overline{f^{[2]}(b)}\right] \\
= & \frac{\Delta \gamma_{1}}{\rho_{2}}\left(\widetilde{\gamma}_{1} g(b) \overline{f(b)}+\widetilde{\gamma}_{2} g(b) \bar{f} \overline{f 2]}(b)\right) \\
= & \frac{\Delta \gamma_{1}}{\rho_{2}}\left(\widetilde{\gamma}_{1}-\widetilde{\gamma}_{2} \frac{\gamma_{1} \lambda+\tilde{\gamma}_{1}}{\gamma_{2} \lambda+\widetilde{\gamma}_{2}}\right) g(a) \overline{f(a)} \\
= & \frac{\lambda \Delta \gamma_{1}}{\gamma_{2} \lambda+\widetilde{\gamma}_{2}} g(b) \overline{f(b)}
\end{aligned}
$$

Dividing both sides of $\Delta \gamma_{1}$, and taking the limit as $\Delta \gamma_{1} \rightarrow 0$, we have

$$
\lambda^{\prime}\left(\gamma_{1}\right)=\frac{\lambda}{\gamma_{2} \lambda+\widetilde{\gamma}_{2}}|f(b)|^{2}
$$

by Theorem 3.3. Using the similar methods of (6), one can prove that (7), (8) and (9) are also true.

(10) Let all the elements of $\omega$ be fixed except $K$. Let $K+R=\left(\begin{array}{ll}\alpha_{1 R} & \widetilde{\alpha}_{1 R} \\ \alpha_{2 R} & \widetilde{\alpha}_{2 R}\end{array}\right)$, with $\operatorname{det}(K+R)=-\rho_{1}$, and

$$
\begin{aligned}
& F(x, K)=\left(f(x, K), f_{1}(K), f_{2}(K)\right)^{T}, \\
& G(x, K)=\left(g(x, K), g_{1}(K), g_{2}(K)\right)^{T}
\end{aligned}
$$

be the normalized eigenvectors corresponding to $\lambda(K)$ and $\lambda(K+R)$, respectively. Then

$$
\begin{aligned}
\lambda(K)\left[\alpha_{1} f(a)-\alpha_{2} f^{[2]}(a)\right] & =\widetilde{\alpha}_{2} f^{[2]}(a)-\widetilde{\alpha}_{1} f(a), \\
\lambda(K+R)\left[\alpha_{1 R} \overline{g(a)}-\alpha_{2 R} \overline{g^{[2]}(a)}\right] & =\widetilde{\alpha}_{2 R} \overline{g^{[2]}(a)}-\widetilde{\alpha}_{1 R} \overline{g(a)}
\end{aligned}
$$

by the boundary condition (2). Using the boundary condition (2)-(4), simple calculation yields 


$$
\begin{aligned}
& {[\lambda(K+R)-\lambda(K)]\langle G, F\rangle} \\
& =\langle\lambda(K+R) G, F\rangle-\langle G, \lambda(K) F\rangle \\
& =[g, \bar{f}]_{a}^{b}+\frac{1}{\rho_{1}} \mathcal{D}_{1}(g) \overline{\mathcal{B}_{1}(f)}-\frac{1}{\rho_{1}} \mathcal{B}_{1}(g) \overline{\mathcal{D}_{1}(f)}+\frac{1}{\rho_{2}} \mathcal{D}_{2}(g) \overline{\mathcal{B}_{2}(f)}-\frac{1}{\rho_{2}} \mathcal{B}_{2}(g) \overline{\mathcal{D}_{2}(f)} \\
& =g^{[2]}(a) \overline{f(a)}-g(a) \overline{f^{[2]}(a)}+\frac{1}{\rho_{1}} \mathcal{D}_{1}(g) \overline{\mathcal{B}_{1}(f)}-\frac{1}{\rho_{1}} \mathcal{B}_{1}(g) \overline{\mathcal{D}_{1}(f)} \\
& =g^{[2]}(a) \overline{f(a)}-g(a) \overline{f^{[2]}(a)}+\frac{1}{\rho_{1}}\left[\widetilde{\alpha}_{2 R} g^{[2]}(a)-\widetilde{\alpha}_{1 R} g(a)\right]\left[\alpha_{1} \overline{f(a)}-\alpha_{2} \overline{f^{[2]}(a)}\right] \\
& -\frac{1}{\rho_{1}}\left[\alpha_{1 R} g(a)-\alpha_{2 R} g^{[2]}(a)\right]\left[\widetilde{\alpha}_{2} \overline{f^{[2]}(a)}-\widetilde{\alpha}_{1} \overline{f(a)}\right] \\
& =\left(-g(a), g^{[2]}(a)\right)\left(\overline{f^{[2]}(a)}, \overline{f(a)}\right)^{T} \\
& +\frac{1}{\rho_{1}}\left(-g(a), g^{[2]}(a)\right)\left(\widetilde{\alpha}_{1 R}, \widetilde{\alpha}_{2 R}\right)^{T}\left(-\alpha_{2}, \alpha_{1}\right)\left(\overline{f^{[2]}(a)}, \overline{f(a)}\right)^{T} \\
& \left.-\frac{1}{\rho_{1}}\left(-g(a), g^{[2]}(a)\right)\left(-\alpha_{1 R},-\alpha_{2 R}\right)^{T}\left(\widetilde{\alpha}_{2},-\widetilde{\alpha}_{1}\right) \overline{\left(f^{[2]}(a)\right.}, \overline{f(a)}\right)^{T} \\
& =\left(-g(a), g^{[2]}(a)\right)\left[E+\frac{1}{\rho_{1}}\left(\widetilde{\alpha}_{1 R}, \widetilde{\alpha}_{2 R}\right)^{T}\left(-\alpha_{2}, \alpha_{1}\right)\right. \\
& \left.-\frac{1}{\rho_{1}}\left(-\alpha_{1 R},-\alpha_{2 R}\right)^{T}\left(\widetilde{\alpha}_{2},-\widetilde{\alpha}_{1}\right)\right]\left(\overline{f^{[2]}(a)}, \overline{f(a)}\right)^{T} \\
& \left.=\left(-g(a), g^{[2]}(a)\right)\left[E+\frac{1}{\rho_{1}}\left(\begin{array}{ccc}
\alpha_{1 R} \widetilde{\alpha}_{2}-\widetilde{\alpha}_{1 R} \alpha_{2} & \widetilde{\alpha}_{1 R} \alpha_{1}-\alpha_{1 R} \widetilde{\alpha}_{1} \\
\alpha_{2 R} \widetilde{\alpha}_{2}-\widetilde{\alpha}_{2 R} \alpha_{2} & \widetilde{\alpha}_{2 R} \alpha_{1}-\alpha_{2 R} \widetilde{\alpha}_{1}
\end{array}\right)\right] \overline{\left(f^{[2]}(a)\right.}, \overline{f(a)}\right)^{T} \\
& =\left(-g(a), g^{[2]}(a)\right)\left[E-(K+R) K^{-1}\right]\left(\overline{f^{[2]}(a)}, \overline{f(a)}\right)^{T} \text {. }
\end{aligned}
$$

Let $R \rightarrow 0$, we get that

$$
[\lambda(K+R)-\lambda(K)](1+\circ(1))=\left(-f(a), f^{[2]}(a)\right)\left[E-(K+R) K^{-1}\right]\left(\overline{f^{[2]}(a)}, \overline{f(a)}\right)^{T},
$$

that is,

$$
\left.\lambda(K+R)-\lambda(K)=\left(-f(a), f^{[2]}(a)\right)\left[E-(K+R) K^{-1}\right] \overline{\left(f^{[2]}(a)\right.}, \overline{f(a)}\right)^{T}+\circ(R) .
$$

Hence (10) holds. The proof of (11) is similar to this.

(12) Let all the elements of $\omega$ be fixed except $p_{0}$, and

$$
\begin{gathered}
F\left(x, p_{0}\right)=\left(f\left(x, p_{0}\right), f_{1}\left(p_{0}\right), f_{2}\left(p_{0}\right)\right)^{T}, \\
G\left(x, p_{0}\right)=\left(g\left(x, p_{0}\right), g_{1}\left(p_{0}\right), g_{2}\left(p_{0}\right)\right)^{T}
\end{gathered}
$$

be the normalized eigenvectors corresponding to $\lambda\left(p_{0}\right)$ and $\lambda\left(p_{0}+\tau\right)$. Then we have 


$$
\begin{aligned}
& {\left[\lambda\left(p_{0}+\tau\right)-\lambda\left(p_{0}\right)\right]\langle G, F\rangle } \\
&=\left[\lambda\left(p_{0}+\tau\right)-\lambda\left(p_{0}\right)\right]\left(\int_{a}^{b} g \bar{f} w d x+\frac{1}{\rho_{1}} g_{1} \overline{f_{1}}+\frac{1}{\rho_{2}} g_{2} \overline{f_{2}}\right) \\
&=\int_{a}^{b} \ell(g) \bar{f} w d x-\int_{a}^{b} g \overline{\ell(f)} w d x+\left[\lambda\left(p_{0}+\tau\right)-\lambda\left(p_{0}\right)\right]\left(\frac{1}{\rho_{1}} g_{1} \overline{f_{1}}+\frac{1}{\rho_{2}} g_{2} \overline{f_{2}}\right) \\
&=\int_{a}^{b}\left[\left(-g^{[2]}\right)^{\prime}+i q_{1} g^{\prime}+p_{1} g\right] \bar{f} d x-\int_{a}^{b} g \overline{\left[\left(-f^{[2]}\right)^{\prime}+i q_{1} f^{\prime}+p_{1} f\right]} d x \\
&+\frac{1}{\rho_{1}} \mathcal{D}_{1}(g) \overline{\mathcal{B}_{1}(f)}-\frac{1}{\rho_{1}} \mathcal{B}_{1}(g) \overline{\mathcal{D}_{1}(f)}+\frac{1}{\rho_{2}} \mathcal{D}_{2}(g) \overline{\mathcal{B}_{2}(f)}-\frac{1}{\rho_{2}} \mathcal{B}_{2}(g) \overline{\mathcal{D}_{2}(f)} \\
&= {\left.\left[\bar{g} \bar{f}^{[2]}-g^{[2]} \bar{f}\right]\right|_{a} ^{b}+\int_{a}^{b}\left[i q_{0}\left(q_{0} g^{\prime}\right)^{\prime}+\left(p_{0}+\tau\right) g^{\prime}-i q_{1} g\right] \bar{f}^{\prime} d x } \\
&-\int_{a}^{b} g^{\prime}\left[-i q_{0}\left(q_{0} \bar{f}^{\prime}\right)^{\prime}+p_{0} \bar{f}^{\prime}+i q_{1} \bar{f}\right] d x+i \int_{a}^{b}\left[q_{1} g^{\prime} \bar{f}+q_{1} g \bar{f}^{\prime}\right] d x \\
&+\frac{1}{\rho_{1}} \mathcal{D}_{1}(g) \overline{\mathcal{B}_{1}(f)}-\frac{1}{\rho_{1}} \mathcal{B}_{1}(g) \overline{\mathcal{D}_{1}(f)}+\frac{1}{\rho_{2}} \mathcal{D}_{2}(g) \overline{\mathcal{B}_{2}(f)}-\frac{1}{\rho_{2}} \mathcal{B}_{2}(g) \overline{\mathcal{D}_{2}(f)} \\
&= \int_{a}^{b}\left(p_{0}+\tau\right) g^{\prime} \bar{f}^{\prime} d x-\int_{a}^{b} p_{0} g^{\prime} \bar{f}^{\prime} d x \\
&= \int_{a}^{b} \tau g^{\prime} \bar{f}^{\prime} d x .
\end{aligned}
$$

It follows from the above results that (12) holds. Using the similar methods of (12), one can prove that (13) and (14) are also true.

\section{Conclusion}

In the present paper, we study the dependence of eigenvalues of third-order differential operators with eigenparameter dependent boundary conditions. It is proved that the eigenvalues of such problem depend not only continuously but also smoothly on the parameter. The dependence of eigenvalues with respect to the data plays an important role in the theory of differential operators. It gives theoretical support for the numerical computation of eigenvalues. Moreover, the properties of monotonicity of eigenvalues with respect to the parameters can be obtained by the derivatives of eigenvalues on the given parameter.

Acknowledgements The authors are grateful to the referees for his/her careful reading and very helpful suggestions which improved and strengthened the presentation of this manuscript.

Open Access This article is distributed under the terms of the Creative Commons Attribution 4.0 International License (http://creativecommons.org/licenses/by/4.0/), which permits unrestricted use, distribution, and reproduction in any medium, provided you give appropriate credit to the original author(s) and the source, provide a link to the Creative Commons license, and indicate if changes were made. 
Author Contributions All authors completed the paper together. All authors read and approved the final manuscript.

Funding This research is partly funded by the National Nature Science Foundation of China (no. 62161045), the Inner Mongolia Autonomous Region University Scientific Research Project (nos. NJZY21570, NJZZ21003), the Natural Science Foundation of Shandong Province (nos. ZR2020QA009, ZR2019MA034).

Availability of data and materials Not applicable.

\section{Declarations}

Conflict of interest The authors declare that they have no competing interests.

Ethics approval Not applicable.

Consent to participate Not applicable.

Consent for publication Not applicable.

Open Access This article is licensed under a Creative Commons Attribution 4.0 International License, which permits use, sharing, adaptation, distribution and reproduction in any medium or format, as long as you give appropriate credit to the original author(s) and the source, provide a link to the Creative Commons licence, and indicate if changes were made. The images or other third party material in this article are included in the article's Creative Commons licence, unless indicated otherwise in a credit line to the material. If material is not included in the article's Creative Commons licence and your intended use is not permitted by statutory regulation or exceeds the permitted use, you will need to obtain permission directly from the copyright holder. To view a copy of this licence, visit http://creativecommons.org/licen ses/by/4.0/.

\section{References}

1. Fulton, C.T.: Two-point boundary value problems with eigenvalue parameter contained in the boundary conditions. Proc. R. Soc. Edinb. Sect. A Math. 77, 293-308 (1977)

2. Olğar, H., Mukhtarov, OSh.: Weak eigenfunctions of two-interval Sturm-Liouville problems together with interaction conditions. J. Math. Phys. 58(4), 388-396 (2017)

3. Binding, P.A., Browne, P.J., Watson, B.A.: Transformations between Sturm-Liouville problems with eigenvalue dependent and independent boundary conditions. Bull. Lond. Math. Soc. 33(6), 749-757 (2001)

4. Guliyev, N.J.: A Riesz basis criterion for Schrödinger operators with boundary conditions dependent on the eigenvalue parameter. Anal. Math. Phys. 10, 2 (2020). https://doi.org/10.1007/ s13324-019-00348-0

5. Binding, P., Browne, P.J., Watson, B.A.: Sturm-Liouville problems with boundary conditions raditionally dependent on the eigenparameter. J. Comput. Appl. Math. 148(1), 147-168 (2002)

6. Mukhtarov, O.S., Aydemir, K.: Eigenfunction expansion for Sturm-Liouville problems with transmission conditions at one interior point. Acta Math. Sci. (Engl. Ser.) 35(3), 639-649 (2015)

7. Mukhtarov, O.S., Aydemir, K.: Two-linked periodic Sturm-Liouville problems with transmission conditions. Math. Methods Appl. Sci. 44, 1-13 (2021)

8. Cai, J., Zheng, Z.: Matrix representations of Sturm-Liouville problems with coupled eigenparameter-dependent boundary conditions and transmission conditions. Math. Methods Appl. Sci. 41(9), 3495-3508 (2018) 
9. Kraft, R.E., Wells, W.R.: Adjointness properties for differential systems with eigenvalue-dependent boundary conditions, with application to flow duct acoustics. J. Acoust. Soc. Am. 58(S1), 913-922 (1975)

10. Li, K., Sun, J., Hao, X., Bao, Q.: Spectral analysis for discontinuous non-self-adjoint singular Dirac operators with eigenparameter dependent boundary condition. J. Math. Anal. Appl. 453(1), 304316 (2017)

11. Guliyev, Namig J.: Schrödinger operators with distributional potentials and boundary conditions dependent on the eigenvalue parameter. J. Math. Phys. 60, 063501 (2019)

12. Uğurlu, E., Bairamov, E.: Spectral analysis of eigenparameter dependent boundary value transmission problems. J. Math. Anal. Appl. 413(1), 482-494 (2014)

13. Kong, Q., Zettl, A.: Eigenvalues of regular Sturm-Liouville problems. J. Differ. Equ. 131, 1-19 (1996)

14. Kong, Q., Wu, H.Y., Zettl, A.: Dependence of the nth Sturm-Lioouville eigenvalue on the problem. J. Differ. Equ. 156(2), 328-354 (1999)

15. Zhu, H., Shi, Y.: Dependence of eigenvalues on the boundary conditions of Sturm-Liouville problems with one singular endpoint. J. Differ. Equ. 263, 5582-5609 (2017)

16. Zhang, M., Sun, J., Zettl, A.: Eigenvalues of limit-point Sturm-Liouville problems. J. Math. Anal. Appl. 419(1), 627-642 (2014)

17. Zhang, M., Li, K.: Dependence of eigenvalues of Sturm-Liouville problems with eigenparameter dependent boundary conditions. Appl. Math. Comput. 378, 125214 (2020). https://doi.org/10. 1016/j.amc.2020.125214

18. Suo, J., Wang, W.: Eigenvalues of a class of regular fourth-order Sturm-Liouville problems. Appl. Math. Comput. 218(19), 9716-9729 (2012)

19. Lv, X., Ao, J., Zettl, A.: Dependence of eigenvalues of fourth-order differential equations with discontinuous boundary conditions on the problem. J. Math. Anal. Appl. 456, 671-685 (2017)

20. Kong, Q.K., Wu, H.Y., Zettl, A.: Dependence of eigenvalues on the problems. Math. Nachr. 188, 173-201 (1997)

21. Uğurlu, E.: Third-order boundary value transmission problems. Turk. J. Math. 43, 1518-1532 (2019)

22. Zhang, M., Wang, Y.: Dependence of eigenvalues of Sturm-Liouville problems with interface conditions. Appl. Math. Comput. 265, 31-39 (2015)

23. Li, K., Sun, J., Hao, X.: Dependence of eigenvalues of $2 n$th order boundary value transmission problems. Bound. Value Probl. 2017(1), 143 (2017)

24. Li, K., Sun, J., Hao, X.: Eigenvalues of regular fourth order Sturm-Liouville problems with transmission conditions. Math. Methods Appl. Sci. 40, 3538-3551 (2017)

25. Bailey, P., Everitt, W., Zettl, A.: The SLEIGN2 Sturm-Liouville code. ACM Trans. Math. Softw. 21, 143-192 (2001)

26. Greenberg, L., Marletta, M.: The code SLEUTH for solving fourth order Sturm-Liouville problems. ACM Trans. Math. Softw. 23(4), 453-493 (1997)

27. Wang, A., Sun, J., Zettl, A.: The classification of self-adjoint boundary conditions: separated, coupled, and mixed. J. Funct. Anal. 255(6), 1554-1573 (2008)

28. Niu, T., Hao, X., Sun, J., Li, K.: Canonical forms of self-adjoint boundary conditions for regular differential operators of order three. Oper. Matrices 14(1), 207-220 (2020)

29. Hao, X.L., Zhang, M.Z., Sun, J., Zettl, A.: Characterization of domains of self-adjoint ordinary differential operators of any order, even or odd. Electron. J. Qual. Theory Differ. Equ. 2017(61), 1-19 (2017)

30. Ma, R., Lu, Y.: Disconjugacy and extremal solutions of nonlinear third-order equations. Commun. Pure Appl. Anal. 13(3), 1223-1236 (2014)

31. Gregus, M.: Third Order Linear Differential Equations (Mathematics and its Applications). Kluwer Academic Publishers, Dordrecht (1987)

32. Uğurlu, E.: Regular third-order boundary value problems. Appl. Math. Comput. 343, 247-257 (2019)

33. Hinton, D.: Deficiency indices of odd-order differential operators. Rocky Mt. J. Math. 8(4), 627640 (1978)

34. Li, K., Bai, Y., Wang, W., Meng, F.: Self-adjoint realization of a class of third-order differential operators with eigenparameter dependent boundary conditions. J. Appl. Anal. Comput. 10(6), 2631$2643(2020)$ 
35. Kong, Q.K., Zettl, A.: Linear ordinary differential equations [M]. In: Agarwal, R.P. (ed.) Inequalities and Applications, vol. 3, pp. 381-397. WSSIAA, Singapore (1994)

36. Zettl, A.: Sturm-Liouville Theory. Mathematical Surveys and Monographs 121. American Mathematical Society, Providence (2005) 